Article

\title{
Analysis of a mechanical vibration filter and amplifier for sounding rocket applications
}

\author{
Adam Dąbrowski ${ }^{1,}$, Szymon Krawczuk ${ }^{2}$ \\ 1 Institute of Mechanics and Machine Design, Faculty of Mechanical Engineering and Ship Technology, \\ Gdańsk University of Technology, ul. Narutowicza 11/12, 80-233 Gdańsk; adadabro@pg.edu.pl \\ 2 Faculty of Mechanical Engineering and Ship Technology, Gdańsk University of Technology, ul. \\ Narutowicza 11/12, 80-233 Gdańsk; szymon.krawczuk@sspg.pl \\ * Correspondence: adadabro@pg.edu.pl; Tel.: +48 583472074
}

Featured Application: Authors are encouraged to provide a concise description of the specific application or a potential application of the work. This section is not mandatory.

\begin{abstract}
Determining the vibration environment is crucial to analyzing a design of any mechanical system, especially such dynamic systems as sounding rockets. Accuracy of measurement using accelerometers could be improved by application of mechanical vibration filtering and amplifying devices. This work presents a theoretical description of a tunable filter and amplifier. Principle of work is provided as well as results from application of the device on a sounding rocket are provided. It is shown that implementation of such devices allowed for enhancing the accuracy of acceleration measurements. Conclusions on future implementations are also provided.
\end{abstract}

Keywords: vibrations; dynamics; sounding rocket; vibration filtering; signal processing; space engineering

\section{Introduction}

Vibration is one of the most important environmental factors that need to be taken into account during the design of a rocket. This is especially true for sounding rockets, characterized by high levels of acceleration (due to high initial thrust) and vibrations [1]. These vehicles are frequently used for scientific purposes providing access to microgravity. Such platform provides up to few minutes of "near-weightlessness" conditions [2]. In these applications, either isolation or suppression of vibrations is of special importance. A number of papers proposed special platforms for that purpose.

The solution by Choi et al. [3] suggests implementation of a notch filter for attitude control. Danowski et al. [4] proposed a suspended system of springs-dampers that additionally prevents thermal energy transfer. An interesting review of various technical solutions for low-cost vibration isolation has been provided by Grosse [5]. Key principles for design of such systems have been summarised by Kramer et al. [6]. The crucial finding is that for proper tuning of such system, a precise information of vibration of the sounding rocket in specific frequencies is required.

The HEDGEHOG REXUS experiment was prepared with its focus on measuring acceleration and vibration conditions (especially eigenfrequencies) inside a REXUS sounding rocket - as a reference for future ground acceptance tests. The REXUS/BEXUS programme is realised under a bilateral Agency Agreement between the DLR and the SNSA. The Swedish share of payload is available to students from other European countries by means of collaboration with the ESA. EuroLaunch, a cooperation between the Esrange Space Center of SSC and the MORABA of DLR, is responsible for managing the campaign and operations of the launch vehicles. Experts from DLR, SSC, ZARM and ESA provide technical support to the student teams throughout the project. REXUS and BEXUS are launched from SSC, Esrange Space Center in northern Sweden [7]. REXUS 
experiments prior to that described herein, included various spaceborne technology demonstrators [8-10]. The preliminary results from HEDGEHOG REXUS project - A sounding rocket experiment on accelerations, vibrations and heat flow were described by Dąbrowski et al. [11].

\section{Materials and Methods}

The purpose of this research was to determine dynamic properties, especially filtering and amplification of mechanical vibrations of a sounding rocket. The model of the device is a lateral cantilever beam with a mass at the end (Figure 1).

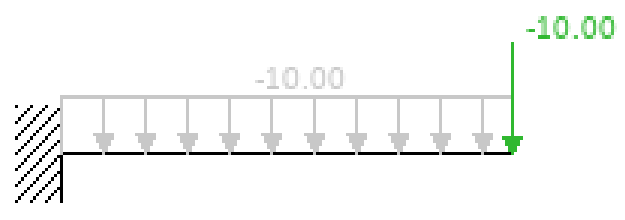

Figure 1. Simple model of a cantilever beam with a mass at the end.

The governing differential equation is:

$$
E \cdot I \frac{d^{2} y(x)}{d x^{2}}=\mathrm{M}(\mathrm{x}),
$$

where: $E$-Young's modulus, $I$ - moment of inertia of sectional area, computed about the neutral axis, $M(x)$ - function describing distribution of the bending moment over the length of the beam. By solving Equation (1), one can calculate the beam deflection as the sum of beam deflection due to beam weight and beam deflection due to mass at the end weight [12]:

$$
y(L)=\delta=\delta_{B}+\delta_{M}=\frac{L^{3}}{E \cdot I}\left(\frac{m_{B}}{8}+\frac{m}{3}\right) g,
$$

where: $\delta$ - beam deflection, $\delta_{B}$ - beam deflection due to beam weight, $\delta_{M}$ - beam deflection due to mass weight, $L$ - length of the beam, $m_{B}$ - mass of the beam, $m$ - mass of the mass at the end, $g$ - gravitational acceleration.

Assuming, linear-elastic behavior of the system (Hooke's law), natural frequency of the model can be calculated [12]:

$$
f_{\text {nat }}=\frac{1}{2 \pi} \sqrt{\frac{k}{m}}=\frac{\sqrt{3}}{2 \pi} \sqrt{\frac{E \cdot I}{L^{3}\left(m+\frac{3}{8} m_{B}\right)^{\prime}}}
$$

This proves that, provided all other parameters (geometrical and material) remain constant, the natural frequency of the device can be modified by changing the length of the beam:

$$
f_{\text {nat }} \propto L^{-\frac{3}{2}},
$$

By moving the mass at the end of the beam along the beam, it is possible to tune the natural frequency of the system.

Assuming a 1 DOF system with a mass $m$, damping $c$, stiffness $k$, and an external excitation force $F$, a dynamical differential equation governing the motion of the system can be formulated:

$$
m \cdot \frac{d^{2} y}{d t^{2}}+c \cdot \frac{d y}{d t}+k \cdot y=F .
$$

Kucharski [13] showed that the solution for the amplitude $A$ of such damped forced vibrations can be calculated as follows: 


$$
A=\frac{F}{k-m \cdot \Omega^{2}+c \cdot \Omega \cdot i^{\prime}}
$$

where: $F-$ Force acting on the system (e.g. weight of the mass), $\Omega$ - frequency of the oscillations of the force, such that $F(t)=F_{0} \mathrm{e}^{i \Omega t}, i$ - imaginary unit. The relation amplitude $A$ vs. external force frequency $\Omega$ shows typical resonant curve (Figure 2 ).

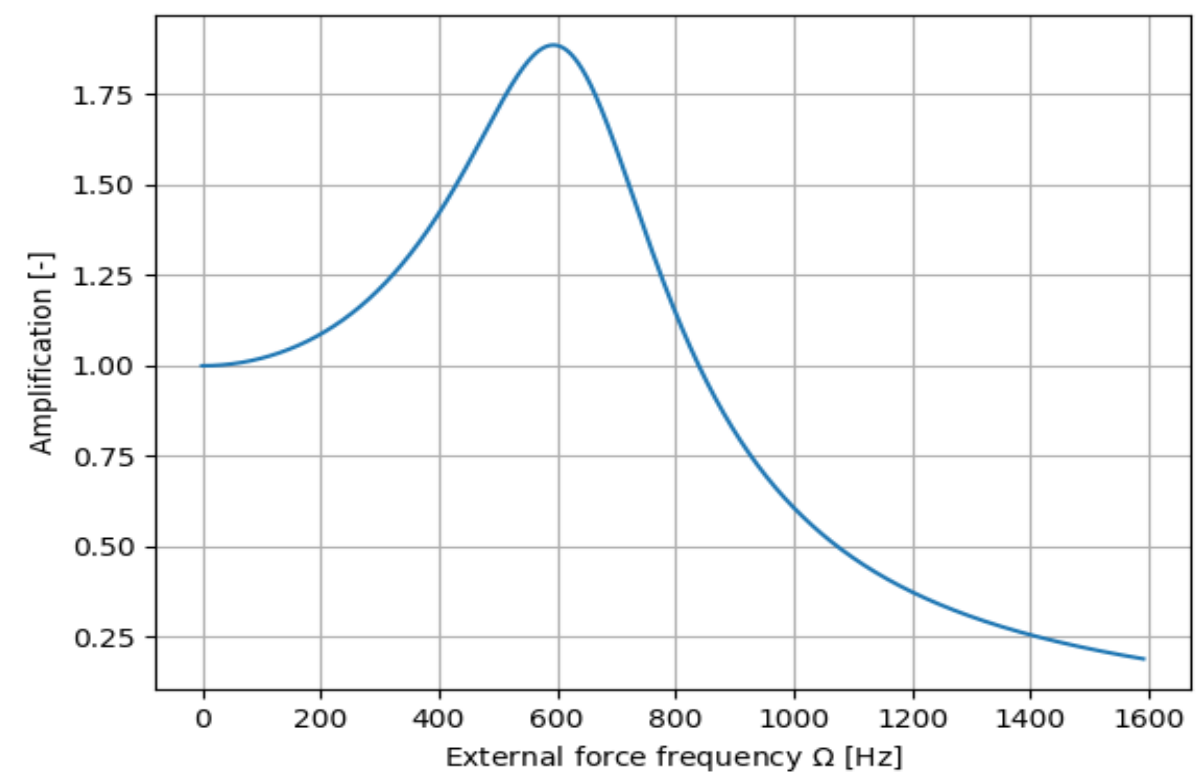

Figure 2. Simple model of a cantilever beam with a mass at the end.

Figure 2 has been calculated for parameters presented in table 1.

Table 1. Parameters of the cantilever beam.

\begin{tabular}{cccc}
\hline Parameter & Symbol & Value & Unit (SI) \\
\hline $\begin{array}{c}\text { Young's modulus } \\
\begin{array}{c}\text { moment of inertia of sectional area } \\
\text { computed about the neutral axis }\end{array}\end{array}$ & $E$ & $6.9 \cdot 10^{10}$ & $\mathrm{~kg} \cdot \mathrm{m}^{-1} \cdot \mathrm{s}^{-2}$ \\
\hline $\begin{array}{c}\text { position of the mass (length of the } \\
\text { beam) }\end{array}$ & $L$ & $5.3 \cdot 10^{-10}$ & $\mathrm{~m}^{4}$ \\
\hline $\begin{array}{c}\text { mass of the mass at the end of the } \\
\text { beam }\end{array}$ & $m$ & $6.5 \cdot 10^{-2}$ & $\mathrm{~m}$ \\
\hline $\begin{array}{c}\text { mass of the beam } \\
\text { gravitational acceleration }\end{array}$ & $m_{B}$ & $6.7 \cdot 10^{-3}$ & $\mathrm{~kg}$ \\
\hline system stiffness (calculated) & $g$ & $4.7 \cdot 10^{-2}$ & $\mathrm{~kg}$ \\
\hline system damping (identified) & $\mathrm{c}$ & $9.8 \cdot 10^{0}$ & $\mathrm{~m} \cdot \mathrm{s}^{-2}$ \\
\hline
\end{tabular}

A design of aluminum 6061-T6 cantilever beams has been prepared (shown in Figure 3). 

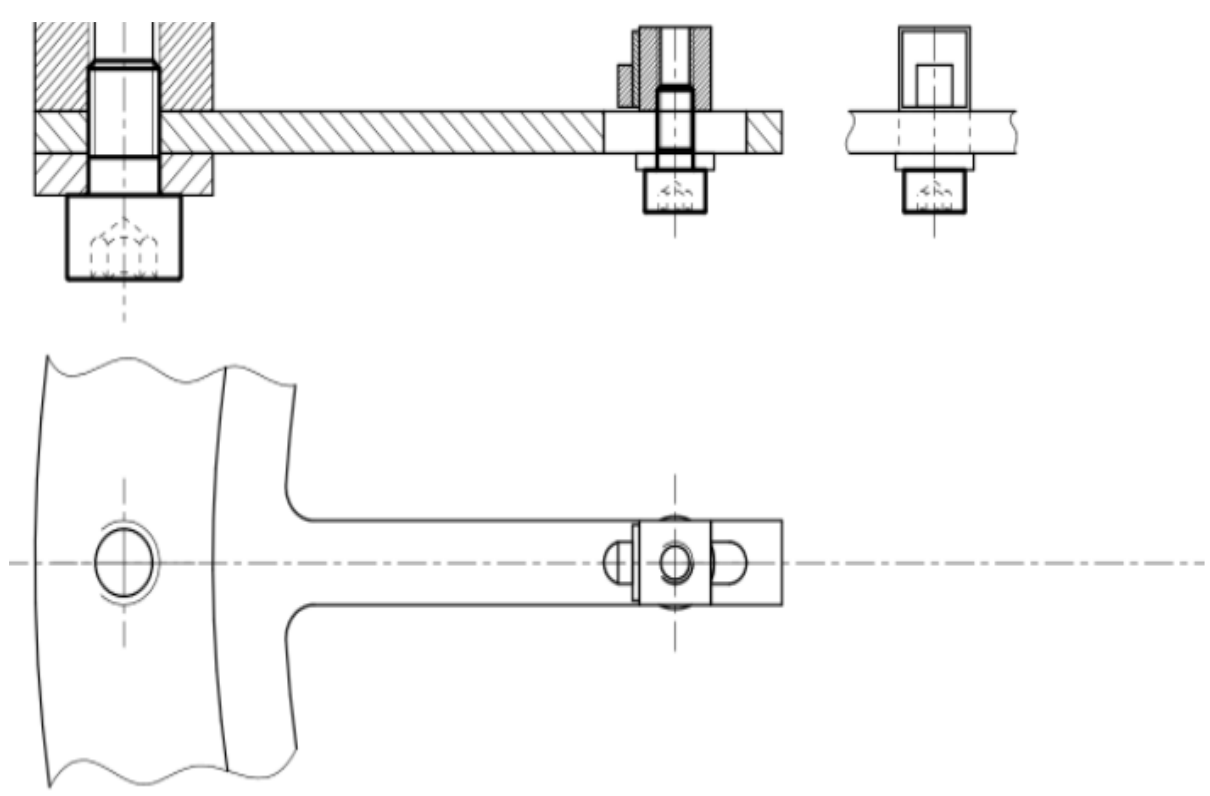

Figure 3. Design of a cantilever beam enabling easy tuning.

At the end of each cantilever beam, a small weight was be mounted by means of M5 Allen Bolt. It was fit together with an oval rather than a hole, which enables one-axial modification of its location. Figure 4 shows the influence of weight location on the principal natural frequency of the system.

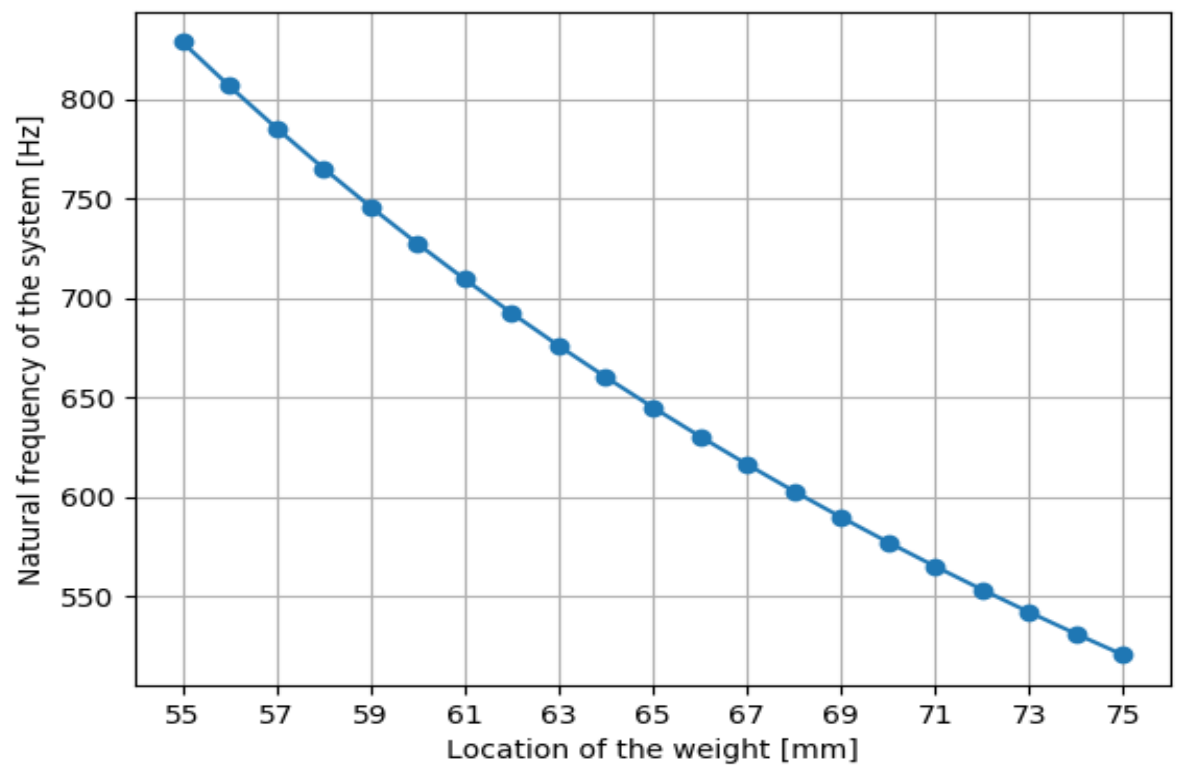

Figure 4. Sensitivity of system eigenfrequency tuning.

An accuracy of positioning of the weight of $1 \mathrm{~mm}$ allowed for $15 \mathrm{~Hz}$ accuracy in eigenfrequency setting.

A system of 10 cantilever beams with tunable eigenfrequencies (see Figure 5) has been placed inside a REXUS sounding rocket. 


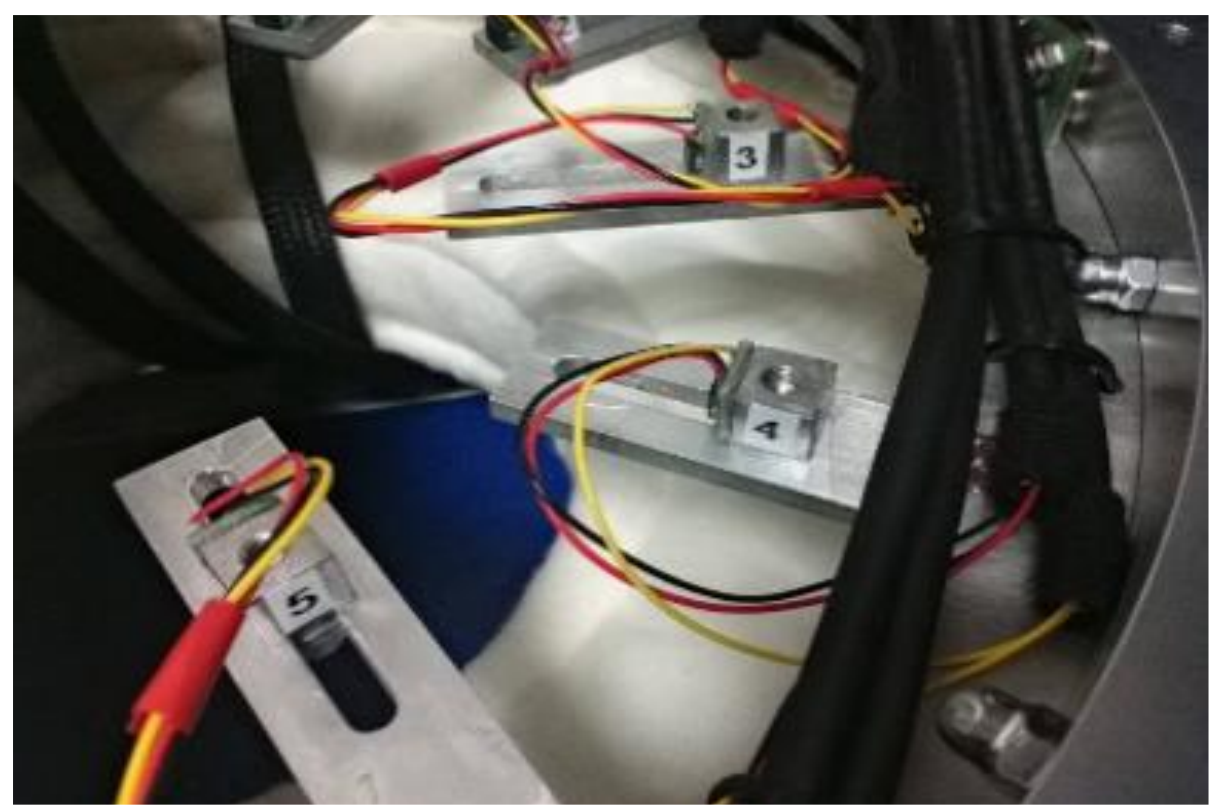

Figure 4. Cantilever beams ( 5 out of 8 ) inside REXUS 25 rocket [11].

\section{Results}

\subsection{Selected eigenfrequencies}

The first longitudinal eigenfrequency of the rocket was identified as $645 \mathrm{~Hz}$, with the use of the modal analysis methodology for sounding rockets developed by Gierse et al. [14]. Several lateral (due to bending of the rocket) eigenfrequencies were also identified, but had not been considered during the design of amplifiers, as the accelerometers used were uniaxial, insensitive in the lateral direction of the rocket. The choice of beams' frequencies was driven by two factors: the desire to cover a typical range of REXUS rockets eigenfrequencies of $300 \mathrm{~Hz}-800 \mathrm{~Hz}$ [14], and focusing on the longitudinal eigenfrequency to verify sensitivity. Table 2 lists eigenfrequencies chosen for the beams.

Table 2. Frequencies of beams in HEDGEHOG REXUS Experiment.

\begin{tabular}{c}
\hline Frequency [Hz] \\
\hline 345 \\
405 \\
\hline 500 \\
\hline 550 \\
\hline 615 \\
\hline 625 \\
\hline 631 \\
\hline 643
\end{tabular}

The reference MEMS accelerometer with $2.5 \mathrm{kHz}$ measurement frequency, $50 \mathrm{~g}$ range and $0.05 \mathrm{~g}$ inaccuracy was placed directly on the inner side of the rocket surface. Measurements were performed during the ascent part of the flight, after motor burnout When positioning the weight cube, with $1 \mathrm{~mm}$ as the distance choice precision, the accuracy of tuning is $15 \mathrm{~Hz}$; however, the impact hammer test enables a more accurate determination of the beam frequency $(1 \mathrm{~Hz})$. At each cube, an uni-axial accelerometer was placed to measure vibrations of the beam (see Fig. 3) with $2.5 \mathrm{kHz}$ measurement frequency and $0.03 \mathrm{~g}$ inaccuracy. 


\subsection{Amplification and filtering}

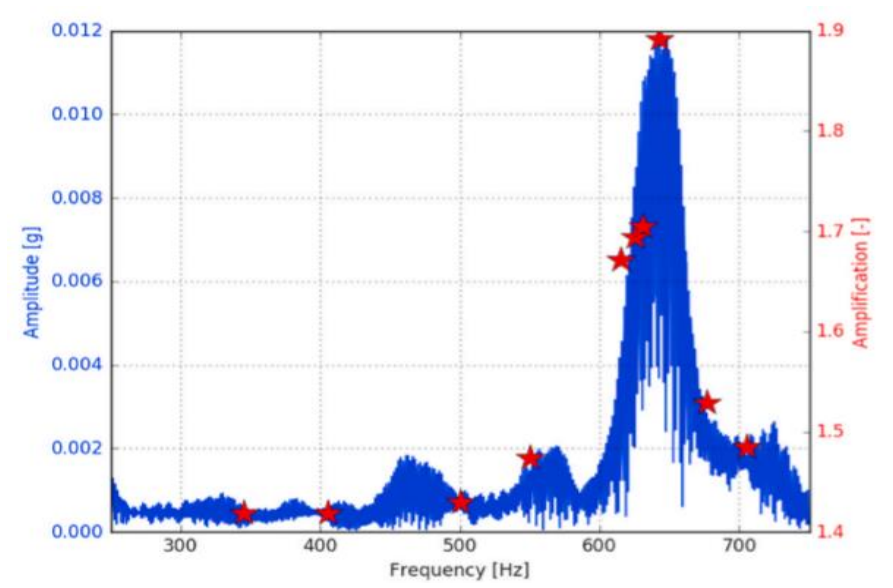

Figure 5. Cantilever amplifications versus the amplitude of vibrations in various frequencies during flight.

Figure 5 shows overall level of vibrations measured with a reference accelerometer (in blue) and the level of amplification of each beam. The level of amplification was defined as:

$$
L o A=\frac{a_{\text {beam }}}{a_{\text {ref }}}
$$

where: $L o A$ - level of acceleration [-], abeam - acceleration at a specific beam accelerometer $\left[\mathrm{m} / \mathrm{s}^{2}\right]$, aref - acceleration at the reference accelerometer $\left[\mathrm{m} / \mathrm{s}^{2}\right]$.

\section{Discussion}

The results show that the device for filtering and amplifying mechanical vibrations worked properly as it matched with the results from the reference accelerometer(see Figure 5). Additionally, it matches directly the predicted characteristics discussed in Chapter 2 (compare Figure 2 and Figure 5).

The application on REXUS rocket provided usability of the device for applications in space technologies. The device applied in such a way could be used to increase accuracy of small vibrations measured by high range accelerometers. Note that values of abeam will have higher accuracy than $a_{r e f}$ by a factor of $L o A$.

\section{Patents}

The device described in this paper is currently pending patent from Polish Patent Office as "A device for amplification and filtering of mechanical vibrations, especially parts of space rockets with tuning possibility".

Author Contributions: Conceptualization, A. Dąbrowski; methodology, A. Dąbrowski.; software, S. Krawczuk.; validation, S. Krawczuk; formal analysis, A. Dąbrowski.; investigation, A. Dąbrowski.; resources, S. Krawczuk.; data curation, A. Dąbrowski.; writing-original draft preparation, A. Dąbrowski.; writing-review and editing, S. Krawczuk.; visualization, S. Krawczuk.; supervision, A. Dąbrowski; project administration, A. Dąbrowski; funding acquisition, S. Krawczuk. All authors have read and agreed to the published version of the manuscript.

Funding: This research received no external funding.

Data Availability Statement: Data from this research can be found here: Dąbrowski, A. (2021). Sounding rocket vibration data [Data set]. Gdańsk University of Technology. https://doi.org/10.34808/8vrj-qs25 .

Acknowledgments: All calculations were carried out at the Academic Computer Center in Gdansk.

Conflicts of Interest: The authors declare no conflict of interest. 


\section{References}

References must be numbered in order of appearance in the text (including citations in tables and legends) and listed individually at the end of the manuscript. We recommend preparing the references with a bibliography software package, such as EndNote, ReferenceManager or Zotero to avoid typing mistakes and duplicated references. Include the digital object identifier (DOI) for all references where available.

Citations and references in the Supplementary Materials are permitted provided that they also appear in the reference list here.

In the text, reference numbers should be placed in square brackets [ ] and placed before the punctuation; for example [1], [1-3] or $[1,3]$. For embedded citations in the text with pagination, use both parentheses and brackets to indicate the reference number and page numbers; for example [5] (p. 10), or [6] (pp. 101-105).

1. Okniński, A., Marciniak, B., Bartkowiak, B., Kaniewski, D., Matyszewski, J., Kindracki, J., \& Wolański, P. Development of the polish small sounding rocket program. Acta Astronaut. 2015, 108, 46-56.

2. Kuhl, R., Roth, M., Binnenbruck, H., Dreier, W., Forke, R., \& Preu, P. The role of sounding rocket microgravity experiments within the German Physical Sciences Programme. In: 17Th ESA Symposium on European Rocket and Balloon Programmes and Related Research 2005, 590, 503-508.

3. Choi, H. D., \& Kim, J. Adaptive notch filter design for bending vibration of a sounding rocket. Proceedings of the Institution of Mechanical Engineers, Part G: Journal of Aerospace Engineering 2001, 215(1), 13-23.

4. Danowski, M. E., Heine, S. N. T., Figueroa-Feliciano, E., Goldfinger, D., Wikus, P., McCammon, D., \& Oakley, P. Vibration isolation design for the micro-x rocket payload. Journal of Low Temperature Physics 2016, 184(3), 597-603.

5. Grosse, J. Thermal and Mechanical Design and Simulation for the first high precision Quantum Optics Experiment on a Sounding Rocket, Doctoral dissertation, Universität Bremen, 2016.

6. Krämer, S., Daab, D. J., Müller, B., Wagner, T., Baader, F., Hessel, J., Pfützenreuter, L. Development and flight-testing of a system to isolate vibrations for microgravity experiments on sounding rockets. In: 17Th ESA Symposium on European Rocket and Balloon Programmes and Related Research 2005, 590, 509-515.

7. REXUS-BEXUS programme. Available online: https://rexusbexus.net/ (accessed on 30 June 2021).

8. H. Mao, T. Sinn, M. Vasile, G. Tibert, Post-launch analysis of the deployment dynamics of a space web sounding rocket experiment, Acta Astronaut. 2016, 127, 345-358.

9. M.R. Roeßner, M.S. Müller, T.C. Buck, A. Koch, Broadband light source for fiberoptic measurement system in spaceborne applications, Acta Astronaut. 2011, 70, 95-99.

10. A. Boesso, A. Francesconi, ARCADE small-scale docking mechanism for microsatellites, Acta Astronaut. 2013, 86, 77-87.

11. Dąbrowski, A., Pelzner, K., Krawczuk, S., Goczkowski, J., \& Elwertowska, A. Preliminary results from HEDGEHOG REXUS project-A sounding rocket experiment on accelerations, vibrations and heat flow. Acta Astronaut. 2020, 177, 80-85.

12. Larson, W. J., Wertz, J. R. Space mission analysis and design, $4^{\text {th }}$ ed. Microcosm, Inc.: Torrance, CA (United States), 2011;

13. Kucharski, T. A method for dynamic response analysis of time-variant discrete systems. Computers \& Structures 2000, 76(4), 545550 .

14. A. Gierse, S. Kramer, € D.J. Daab, J. Hessel, F. Baader, B.S. Müller, T. Wagner, G. Gdalewitsch, E. Plescher, L. Pfützenreuter, Experimental in-flight modalanalysis of a sounding rocket structure In: Proceedings of the 21st ESA Symposium on Rocket $\mathcal{E}$ Balloon Programmes and Related Research, 2013, ESA SP-721. 